\title{
Gastroesophageal reflux disease: from heartburn to Barrett esophagus, and beyond
}

\author{
Marco G. Patti ${ }^{1}$. Francisco Schlottmann ${ }^{1}$
}

Received: 17 August 2018 / Accepted: 18 August 2018 / Published online: 24 August 2018

(c) Italian Society of Surgery (SIC) 2018, corrected publication September 2018

The prevalence of gastroesophageal reflux disease in the western world has been increasing dramatically in the last 2 decades. This disease affects the quality of life of millions of individuals, causing a major burden on the health care system.

While in the past the focus was mostly on the esophageal symptoms such as heartburn and regurgitation, today it is clear that GERD can cause extra-esophageal problems such as hoarseness, cough and chest pain. Some of these patients are seen today in cardiology clinics, ENT clinics and pulmonary clinics. Finally, GERD can have fatal consequences not only through the development of Barrett esophagus and eventually adenocarcinoma, but also being one of the factors that causes pulmonary fibrosis and rejection after lung transplantation.

In this symposium, experts in the treatment of gastroesophageal reflux disease discuss important topics that are of key importance for the evaluation and treatment of patients with this disease. The pathophysiology of the disease has been described, underlying how the medical treatment only decreases the production of acid but does not stop reflux trough an incompetent lower esophageal sphincter. On the other hand, a properly constructed fundoplication restores the competence of the sphincter, therefore, blocking any type of reflux.

Patients with gastroesophageal reflux disease (GERD) may present with a variety of symptoms, including heartburn, regurgitation, dysphagia, chronic cough, laryngitis, or even asthma. The diagnostic work-up of these patients should

The original version of this article was revised: Due to author name update.

Marco G. Patti

marco_patti@med.unc.edu

Francisco Schlottmann

fschlottmann@hotmail.com

1 University of North Carolina, Chapel Hill, NC, USA include a symptomatic evaluation, upper endoscopy, barium swallow, high-resolution manometry, and ambulatory $\mathrm{pH}$ monitoring. Once a proper diagnosis of GERD is achieved, anti-reflux surgery is an excellent option for patients with partial control of symptoms with medication, for patients who do not want to be on long-term medical treatment (compliance/ cost), or when complications of medical treatment occur.

A properly executed laparoscopic fundoplication controls esophageal and extra-esophageal symptoms and avoids lifelong medical therapy. The key technical elements have been clearly illustrated, to obtain optimal and long-lasting results.

We have also discussed the treatment of GERD in the obese patients underlying the role of the increased transdiaphragmatic pressure gradient. In morbidly obese patients with GERD a Nissen fundoplication and a sleeve gastrectomy should be avoided, and a Roux-en-Y gastric bypass should be chosen. This procedure, in fact, controls acid and bile reflux in addition to causing weight loss and resolving or improving other co-morbidities such as diabetes mellitus.

We have also discussed the possibility of a progression to Barrett esophagus, with transformation of the normal esophageal squamous mucosa into a simple columnar epithelium secondary to the chronic injury produced by recurrent reflux episodes.

We do hope that the readership of Updates in Surgery will find the symposium simple to read and useful for the treatment of their patients.

\section{Compliance with ethical standards}

Conflict of interest Marco G. Patti and Francisco Schlottmann declare that they have no conflict of interest.

Research involving human participants and/or animals This article does not contain any studies with human participants or animal performed by any of the authors.

Informed consent For this type of study formal consent is not required. 